\title{
Axonal degeneration in paraplegin- deficient mice is associated with abnormal mitochondria and impairment of axonal transport
}

\author{
Fatima Ferreirinha, ${ }^{1,2}$ Angelo Quattrini, ${ }^{3}$ Marinella Pirozzi, ${ }^{1}$ Valentina Valsecchi, ${ }^{1}$ \\ Giorgia Dina, ${ }^{3}$ Vania Broccoli, ${ }^{1}$ Alberto Auricchio, ${ }^{1}$ Fiorella Piemonte, ${ }^{4}$ Giulia Tozzi, ${ }^{4}$ \\ Laura Gaeta, ${ }^{4}$ Giorgio Casari, ${ }^{5}$ Andrea Ballabio, ${ }^{1,6}$ and Elena I. Rugarli ${ }^{1}$
}

${ }^{1}$ Telethon Institute of Genetics and Medicine, Naples, Italy

${ }^{2}$ Unidade de Investigação Genética e Epidemiológica de Doenças Neurológicas-Instituto de Biologia Molecular e Celular (UnIGENe-IBMC) and Instituto de Ciências Biomédicas Abel Salazar (ICBAS), University of Porto, Porto, Portugal

${ }^{3}$ Department of Neurology, University Vita-Salute, San Raffaele Scientific Institute, Milan, Italy

${ }^{4}$ Molecular Medicine Unit, Department of Neurosciences, Children's Hospital Bambino Gesù, Rome, Italy

${ }^{5}$ Human Molecular Genetics Unit, San Raffaele Scientific Institute, Milan, Italy

${ }^{6}$ Medical Genetics, Department of Pediatrics, Federico II University, Naples, Italy

In several neurodegenerative diseases, axonal degeneration occurs before neuronal death and contributes significantly to patients' disability. Hereditary spastic paraplegia (HSP) is a genetically heterogeneous condition characterized by selective degeneration of axons of the corticospinal tracts and fasciculus gracilis. HSP may therefore be considered an exemplary disease to study the local programs mediating axonal degeneration. We have developed a mouse model for autosomal recessive HSP due to mutations in the SPG7 gene encoding the mitochondrial ATPase paraplegin. Paraplegin-deficient mice are affected by a distal axonopathy of spinal and peripheral axons, characterized by axonal swelling and degeneration. We found that mitochondrial morphological abnormalities occurred in synaptic terminals and in distal regions of axons long before the first signs of swelling and degeneration and correlated with onset of motor impairment during a rotarod test. Axonal swellings occur through massive accumulation of organelles and neurofilaments, suggesting impairment of anterograde axonal transport. Retrograde axonal transport is delayed in symptomatic mice. We speculate that local failure of mitochondrial function may affect axonal transport and cause axonal degeneration. Our data suggest that a timely therapeutic intervention may prevent the loss of axons.

J. Clin. Invest. 113:231-242 (2004). doi:10.1172/JCI200420138.

\section{Introduction}

Hereditary spastic paraplegia (HSP) comprises a heterogeneous group of neurodegenerative diseases characterized by progressive weakness, spasticity, and loss of vibratory sense in the lower limbs. These disorders are classified according to the mode of inheritance - which can be autosomal dominant, autosomal recessive, or $\mathrm{X}$-linked - and whether they manifest as "pure" or "complicated" forms $(1,2)$. Besides lower limb spasticity, the complicated forms are characterized by the occurrence of other symptoms, including mental retardation, cortical

Received for publication September 26, 2003, and accepted in revised form November 4, 2003.

Address correspondence to: Elena I. Rugarli, Telethon Institute of Genetics and Medicine, Via P. Castellino 111, Naples 80131, Italy. Phone: 39-081-6132221; Fax: 39-081-5609877;

E-mail: rugarli@tigem.it.

Vania Broccoli's present address is: Stem Cell Research Institute, San Raffaele Scientific Institute, Milan, Italy.

Conflict of interest: The authors have declared that no conflict of interest exists.

Nonstandard abbreviations used: hereditary spastic paraplegia (HSP); ATPase associated with a variety of cellular activities (AAA). and cerebellar atrophy, optic neuropathy, and peripheral neuropathy $(3,4)$. The neuropathologic hallmark of HSP is degeneration of axons of the corticospinal tracts and the fasciculus gracilis, with fairly good preservation of the neuronal cell bodies (4-6). However, very few autoptic studies are available, and the mechanisms underlying axonal degeneration and the tissue specificity of the phenotype are poorly understood.

Loss-of-function mutations in the SPG7 gene were found in an autosomal recessive form of HSP complicated by optic atrophy, sensorimotor peripheral neuropathy, and cerebellar and cortical atrophy (7). SPG7 encodes a protein named paraplegin, consisting of 795 amino acids, that is highly homologous to two yeast mitochondrial proteins, Yta10p (Afg3p) and Yta12p (Rca1p) (8). These proteins belong to a large family of highly conserved ATPases characterized by the presence of the AAA ( $\underline{\text { ATPases }}$ Associated with a variety of cellular Activities) domain and implicated in a variety of cellular processes, including vesicular transport, organelle biogenesis, microtubule rearrangement, and protein degradation $(9,10)$. In addition to the AAA domain, Yta10p, Yta12p, and paraplegin harbor a binding site 
for divalent metal ions characteristic of metal-dependent proteases. In yeast mitochondria, Yta10p and Yta12p assemble in multiple copies to form the m-AAA protease, a high-molecular-weight complex located in the mitochondrial inner membrane that exposes catalytic sites to the mitochondrial matrix (8). This complex mediates ATP-dependent degradation of mitochondrial inner membrane proteins that might accumulate in case of an imbalance between the expression of respiratory chain subunits encoded by the nuclear and the mitochondrial DNA. The complex also exerts crucial functions during mitochondrial biogenesis by affecting the splicing of transcripts of mitochondrial genes encoding respiratory chain subunits and by chaperoning the posttranslational assembly of respiratory complexes and the ATP synthase (11).

Paraplegin localizes to mitochondria, and in some cases muscle biopsies from severely affected patients bearing paraplegin mutations showed signs of mitochondrial function impairment, such as ragged-red fibers and cytochrome $c$ oxidase-deficient fibers $(7$, 12). Recently, a mutation in mitochondrial heat shock protein 60 has been identified in a family with pure autosomal-dominant HSP (13), suggesting that some forms of HSP result from impairment of mitochondrial protein quality control.

To understand the mechanism through which the loss of a ubiquitous mitochondrial protein leads to the selective degeneration of a subset of axons, we generated and characterized paraplegin-deficient mice. These animals display retrograde degeneration of long axons, thus recapitulating the human disease. Ultrastructural studies of affected axons show that lack of paraplegin leads to the appearance of giant, morphologically abnormal mitochondria specifically in synaptic terminals and distal regions of long axons, followed by a defect in axonal transport and axonal degeneration.

\section{Methods}

Gene targeting. The gene targeting vector was constructed by replacing a 2 -kb XhoI-PstI fragment containing the first two coding exons of mouse paraplegin with the selectable neomycin resistance gene flanked by two lox sites. This vector contained $2 \mathrm{~kb}$ of $5^{\prime}$ and $4 \mathrm{~kb}$ of $3^{\prime}$ homologous genomic sequences, and a thymidine kinase cassette for negative selection. Following electroporation of ES cells, positive selection with G418, and negative selection with ganciclovir, two clones positive for homologous recombination were identified by Southern analysis using EcoRV digestion. The $5^{\prime}$ probe consisted of a 400-bp BamHI-NcoI fragment located outside the homology region, while a PCR fragment corresponding to exon 3 was used as a $3^{\prime}$ probe. Homologous recombinant clones were injected into C57BL/6 blastocysts. The resulting chimeras were then mated to C57BL $/ 6$ females to obtain germline transmission. All the studies described have been performed in a mixed C57BL/6-129Sv genetic background. We confirmed that the phenotype is the same in C57BL/6 and $129 \mathrm{~Sv}$ pure genetic backgrounds. The genotype of mice was identified by PCR using specific primers for either the targeted or the WT alleles. Studies were done in accordance with guidelines of the Institutional Animal Care and Use Committee, Cardarelli Hospital, Naples, Italy. RT-PCR was performed on total embryo cDNA using primers that amplify a region of the mouse Spg7 cDNA spanning exons 3 and 4 that are not deleted in the targeted allele. Oligonucleotide sequences are available upon request.

Antibodies and Western analysis. The cDNA region corresponding to amino acids 50-307 of mouse paraplegin was subcloned into the PQE30 vector (QIAGEN $\mathrm{GmbH}$, Hilden, Germany). The fusion protein was purified under denaturing conditions by nickel-nitrilotriacetic acid metal affinity chromatography and used to produce polyclonal antibodies in rabbits. The antiserum was purified on an acetone powder of tissue extracts derived from Spg $7^{->}$mice (14) and used 1:6,000 in Western blot analysis.

Rotarod analysis. Motor performance was evaluated with a Rota-Rod apparatus (accelerating model; Ugo Basile, Varese, Italy). Groups of 14 Spg $7^{-/-}$and 14 agematched heterozygous mice were analyzed at 2, 4, 7, 12 , and 17 months of age. In all cases mice were placed on the accelerating rod at a starting speed of $4 \mathrm{rpm}$, reaching a final speed of $40 \mathrm{rpm}$ in 5 minutes. At 2 months of age, mice were tested for three trials per day for two consecutive days, while four trials per day were given to mice at 4, 7,12 and 17 months of age. At 12 months and 17 months of age, mice were tested for only 1 day. The animals were allowed to stay on the rod for a maximum of 600 seconds and the time of hold on the rod was scored. The results were analyzed by a two-factor-with-replication ANOVA for genotype and trial.

Isolation of mitochondria, luminometric assay of ATP, and respiratory chain activities. Spinal cord mitochondria were isolated as previously described (15), without the use of nagarse. For assay of ATP synthesis, independent experiments were performed with 7-monthold $(n=3)$, 17-month-old $(n=5)$, and 23-month-old mice $(n=6)$, in each case testing one $\operatorname{Spg} 7^{+/+}$and one Spg $7^{-/}$mouse. Within each experiment, samples were assayed in duplicate or triplicate. Approximately $25 \mu \mathrm{g}$ of mitochondrial protein was resuspended in buffer (250 $\mathrm{mM}$ sucrose, $20 \mathrm{mM}$ Mops, $1 \mathrm{mM}$ EDTA, $5 \mathrm{mM}$ inorganic phosphate, $1 \mathrm{mM} \mathrm{ADP}$, and $0.1 \% \mathrm{BSA}$ ) and incubated for 30 minutes at $37^{\circ} \mathrm{C}$ in the absence of substrate or in the presence of $5 \mathrm{mM}$ pyruvate and 1 $\mathrm{mM}$ L-malate. ATP concentration was determined using a luminometric method as described (16). Mitochondrial ATP was calculated by subtracting the basal ATP value obtained in the absence of substrate from the ATP value measured in the presence of pyruvate/malate. Data were expressed as percentage of the mean WT value. Statistical analysis of the results was performed with a paired $t$ test. Spectrophotometric measurements of respiratory chain enzyme activities were carried out as reported (17). 

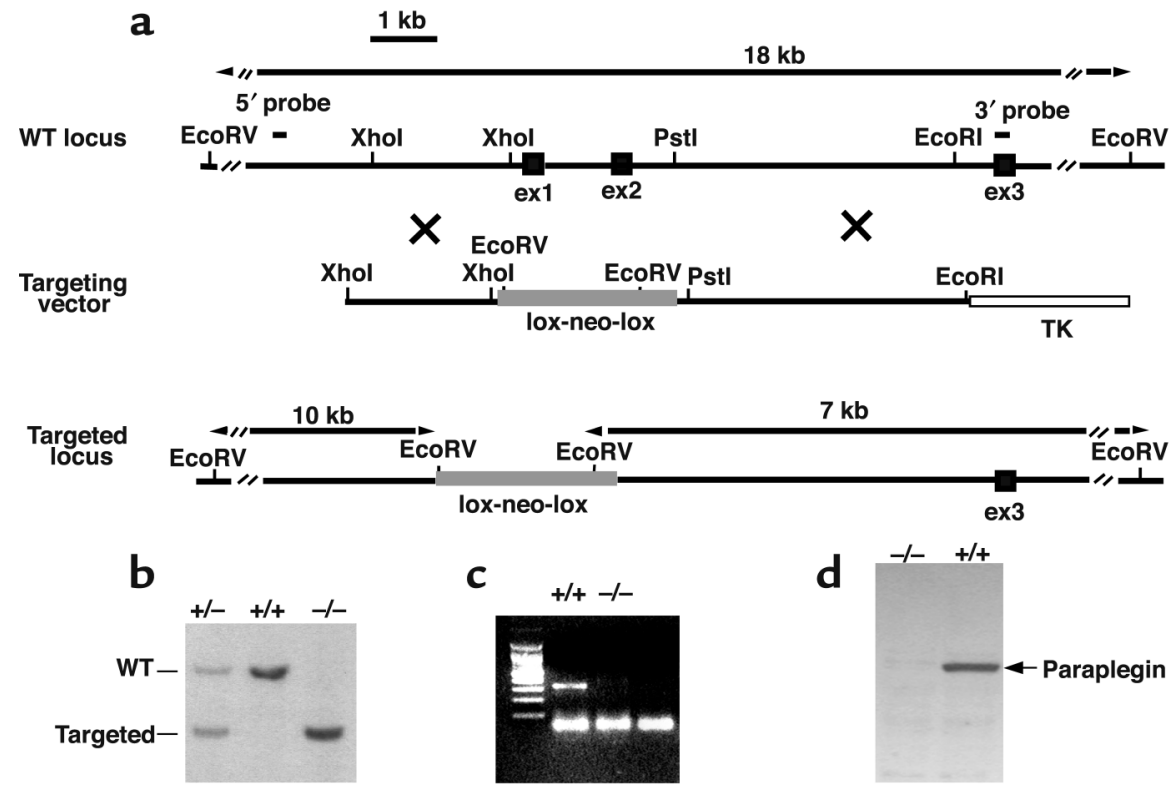

\section{Figure 1}

Strategy of the Spg7 knockout. (a) The top diagram shows the WT genomic map comprising the first three Spg7 exons (ex), the position of the two external probes, and the EcoRV sites used for Southern analysis. On the bottom is a schematic representation of the targeting construct and the resulting targeted locus. Following homologous recombination, the insertion disrupted part of the promoter region and the first two exons of the gene, comprising the initiation methionine codon and most of the mitochondrial leader sequence. (b) Southern analysis of the first litter using the $5^{\prime}$ probe. The WT fragment is $18 \mathrm{~kb}$, whereas the fragment corresponding to the targeted locus is $10 \mathrm{~kb}$. (c) RT-PCR analysis on total embryo cDNA using oligonucleotides spanning exons 3 and 4, which were not targeted by the deletion, demonstrates that the Spg7 transcript is lacking in knockout mice. (d) Western blot analysis on mitochondrial proteins isolated from spinal cords of WT and knockout mice shows the absence of paraplegin in $\mathrm{Spg} \mathrm{7}^{-/-}$animals.

Neuropathology and morphometry. Semithin and ultrathin morphological analyses were conducted as described $(18,19)$. Age-matched $S p g 7^{-/-}$and control mice were anesthetized intraperitoneally with avertin and perfused with $4 \%$ paraformaldehyde in PBS. Cross sections were obtained from the optic nerve, spinal cord, spinal roots, and sciatic nerve. The spinal cords were removed, postfixed in $0.12 \mathrm{M}$ phosphate buffer/ $2 \%$ glutaraldehyde, and divided into three parts (cervi$\mathrm{cal}$, thoracic, and lumbar). They were then sectioned transversely into 2 -mm blocks, postfixed with osmium tetroxide, and embedded in Epon (Fluka, Buchs SG, Switzerland). Cross sections were cut from the cervical and lumbar blocks. Abnormal axons were counted in sections from cervical and lumbar spinal cord of Spg $7^{-/}$ and control mice at different ages. An average of 12 microscopic fields per mouse was analyzed to cover the entire cross section of the spinal cord. Images were collected using a color video camera and the number of damaged axons was quantified. Counting of the axons was performed with the genotype of the mice unknown to the experimenter. For quantitative ultrastructural analyses, blocks of tissue were selected for electron microscopy after light microscopy examination of semithin sections. All ultrastructural analyses were performed in a blinded and nonbiased manner on photographs captured using an electron microscope (magnification $\times 3,000)$. For all mice, nonoverlapping photographs were taken from the lumbar anterolateral columns. An average of 435 axons per mouse was analyzed (see Table 2). Neurofilament densities were quantitatively evaluated in $S p g 7^{-/-}$and control sciatic nerve fibers by overlaying a transparent square profile onto electron micrographs at a final magnification of $\times 60,000$. Each square represented a $0.339-\mu \mathrm{m}^{2}$ region of the axoplasm, not containing membrane-bound organelles. Three axons in true cross sections were randomly selected for $\operatorname{Spg} 7^{--}(n=2)$ and control $(n=2)$ mice. The number of neurofilaments present in 3-5 squares/axon was counted. Determination of cytochrome $c$ oxidase activity was performed as described (20) on fresh-frozen muscle of 13-month-old $(n=4$ for each genotype), 18-month-old ( $n=2$ for each genotype), and 22 -month-old mice ( $n=4$ for each genotype). $\mathrm{H} \& \mathrm{E}$ staining was done using standard techniques. Immunoperoxidase staining using anti-H- neurofilament antibody (Chemicon International, Temecula, California, USA) was performed on frozen sections of the hindpaws of two Spg $7^{-1-}$ and one control mouse at 8 months of age using standard techniques.

Neurotracer experiments. Seventeen-month-old Spg $7^{-1}$ and $\operatorname{Spg} 7^{+/-}$control mice ( $n=3$ for each genotype) were anesthetized with avertin, and an incision 2 - to $3-\mathrm{mm}$ long was made in the skin to expose the gastrocnemius muscle. A total volume of $3 \mu \mathrm{l}$ of $1 \%$ cholera toxin subunit B (Alexa Fluor 488 conjugate; Molecular Probes Inc., Eugene, Oregon, USA) in $\mathrm{H}_{2} \mathrm{O}$ was injected into three different sites of the muscle. Mice were sacrificed 
Table 1

Quantification of axonal changes per section in the spinal cord of Spg $7^{-/-}$and WT littermates at different ages

\begin{tabular}{|c|c|c|c|c|c|c|c|c|}
\hline \multirow[t]{2}{*}{ Age } & \multirow[t]{2}{*}{ Genotype } & \multirow[t]{2}{*}{$n$} & \multicolumn{2}{|c|}{ Anterolateral funiculi } & \multicolumn{2}{|c|}{ Fasciculus gracilis } & \multicolumn{2}{|c|}{ CST } \\
\hline & & & $\mathrm{C}$ & $\mathrm{L}$ & $\mathrm{C}$ & $\mathrm{L}$ & C & L \\
\hline \multirow[t]{2}{*}{4.5 months } & $+/+$ & 3 & 0 & 0 & 0 & 0 & 0 & 0 \\
\hline & $-/-$ & 4 & 0 & 0 & 0 & 0 & 0 & 0 \\
\hline \multirow[t]{2}{*}{8 months } & $+/+$ & 6 & 0 & 0 & 0 & 0 & 0 & 0 \\
\hline & $-/-$ & 6 & 0 & $5.7 \pm 1.9^{\mathrm{A}}$ & $0.5 \pm 0.5$ & 0 & 0 & 0 \\
\hline \multirow[t]{3}{*}{15 months } & $+/+$ & 3 & 0 & 0 & 0 & 0 & 0 & 0 \\
\hline & $-/-$ & 5 & 0 & $27 \pm 8.2^{\mathrm{B}}$ & $11.6 \pm 3.6^{\mathrm{B}}$ & 0 & 0 & 0 \\
\hline & & & & $5.8 \pm 1.3^{\mathrm{A}}$ & $3.2 \pm 2.4$ & & & \\
\hline \multirow[t]{4}{*}{29 months } & $+/+$ & 3 & $0.7 \pm 1.2$ & $1 \pm 1.7$ & $0.7 \pm 1.2$ & 0 & 0 & $0.3 \pm 0.6$ \\
\hline & & & & $1.3 \pm 0.6$ & $0.7 \pm 1.2$ & & & \\
\hline & $-/-$ & 3 & $1.3 \pm 1.2$ & $27.3 \pm 1.5^{C}$ & $10.7 \pm 3.8$ & 0 & 0 & $2.3 \pm 1.5$ \\
\hline & & & & $29.3 \pm 3.8^{\mathrm{B}}$ & $15.3 \pm 4.5$ & & & $0.3 \pm 0.6$ \\
\hline
\end{tabular}

Axonal swellings are indicated in normal text, axonal degenerations in bold. CST, corticospinal tract; C, cervical spinal cord; L, lumbar spinal cord. Statistical analysis was performed using the Student's $t$ test. ${ }^{A} P<0.001 ;{ }^{B} P<0.01 ; C P<0.0001$.

48 hours later and the lumbar enlargement of the spinal cord was harvested and postfixed overnight with $4 \%$ paraformaldehyde in PBS. Tissue was cryoprotected in $30 \%$ sucrose solution and sectioned transversely $(30 \mu \mathrm{m})$ on a cryostat. All sections were collected starting cranially in a sequential order. The number of fluorescent motor neurons in the cranial, middle, and caudal regions of the lumbar enlargement was identified for each animal after allocating the same number of sections to each region. Statistical analysis was performed using an unpaired Student's $t$ test.

Accession number. The GenBank accession number for the $\operatorname{Spg} 7$ mouse cDNA is AF512565.

\section{Results}

Progressive motor impairment in paraplegin-deficient mice. We isolated the murine homologue of $S P G 7$ by searching EST databases and screening a mouse embryo cDNA library using the human SPG7 cDNA as a probe. These experiments led us to the assembly of a $2.6-\mathrm{kb}$ cDNA encoding a protein of 781 amino acids. Mouse paraplegin is $88 \%$ identical to its human counterpart and has a conserved-domain composition. The mitochondrial localization of mouse paraplegin was confirmed by expressing Spg7 cDNA in COS-7 cells and by performing immunofluorescence studies using a specific polyclonal antibody (not shown). In order to a
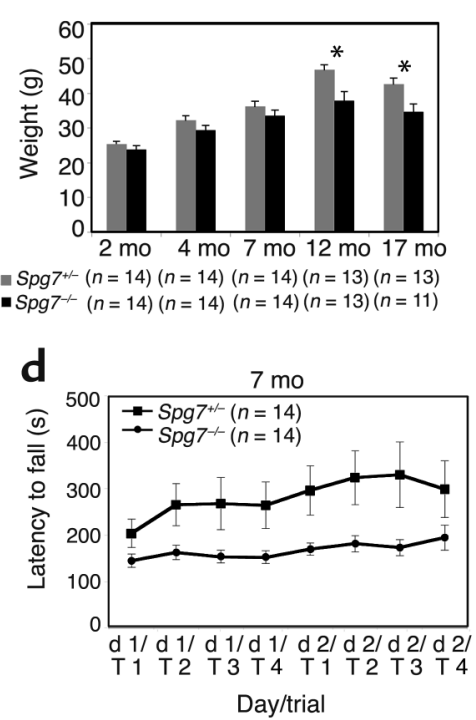

b
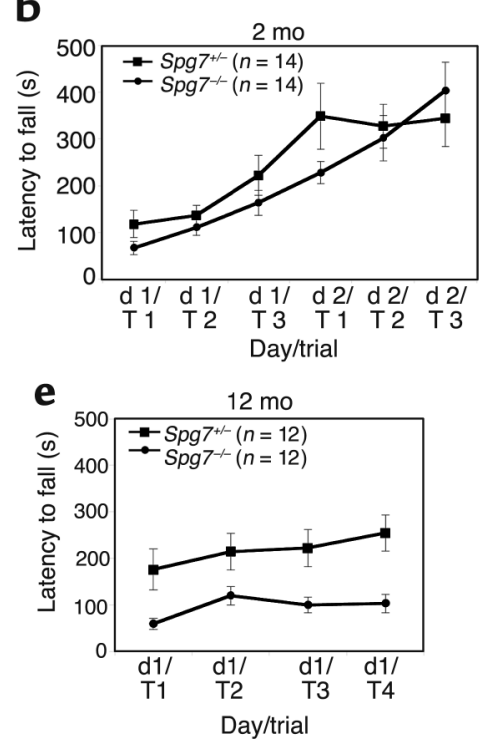

C
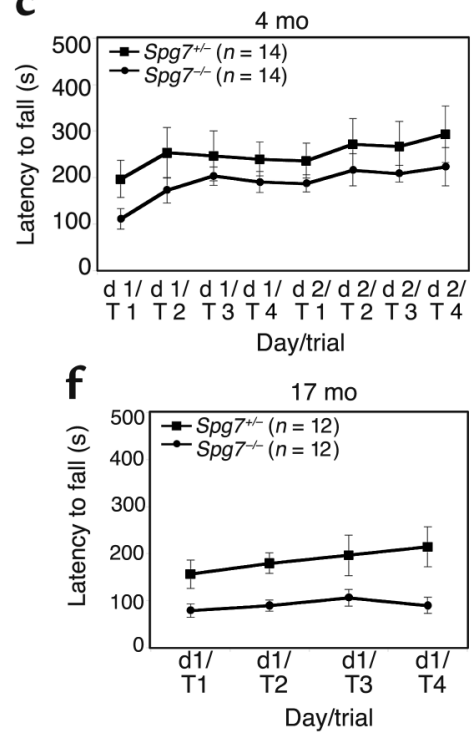

Figure 2

Behavioral phenotype of paraplegin-deficient mice. (a) The weights of Spg $7^{-1-}$ mice are reduced compared with control mice beginning at 12 months of age ( $\left.{ }^{*} P<0.01\right)$. Performance of $S p g 7^{+/-}$and $S p g 7^{-/-}$mice on an accelerating rotating rod apparatus at 2 months $(\mathbf{b}), 4$ months $(\mathbf{c})$, 7 months (d), 12 months (e), and 17 months (f). Spg $7^{-1-}$ animals showed impaired performance compared with heterozygous age-matched animals beginning at 4 months of age. Repeated-measures ANOVA confirmed a significant genotype effect (heterozygous versus knockout) at 4 months of age $(P<0.005)$ and at 7,12 , and 17 months of age $(P<0.00001)$. T, trial. 


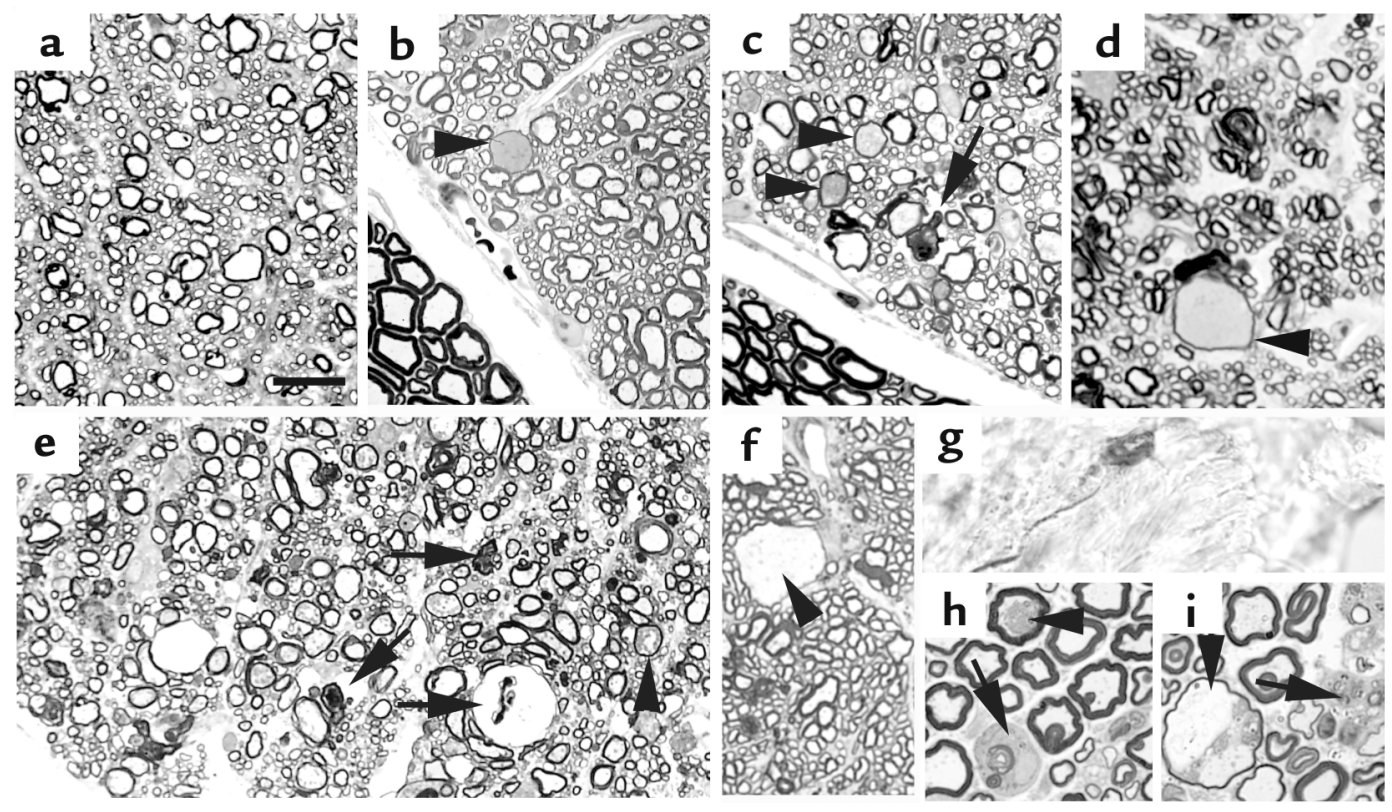

\section{Figure 3}

Paraplegin-deficient mice show axonal swelling and degeneration. Semithin transverse sections of the spinal cord of Spg7-/- mice at different ages. At 4 months, the spinal cord appears normal (a). At 8 months, sporadic swollen axons are detected (arrowhead) (b). Several swollen fibers (arrowheads) and degenerating fibers (arrows) are evident in the anterolateral funiculi (c) and dorsal columns (d) at 15 months. Note normal ventral roots in $\mathbf{b}$ and $\mathbf{c}$. In a 24-month-old Spg7-/- mouse, besides swelling (arrowheads) and degeneration (arrows), the density of myelinated axons is decreased, indicating significant axonal loss (e). (f) Semithin section of the optic nerve of a 19-month-old Spg7-/- mouse showing a swollen axon (arrowhead). (g) Immunostaining for neurofilaments in the derma of the distal hindlimbs of an 8-month-old Spg7-1mouse showing an axonal spheroid. Axonal swelling (arrowhead) and degeneration (arrows) in the sciatic nerve of Spg $7^{-/-}$mice at 19 months (h) and 24 months (i). Bar represents $40 \mu \mathrm{m}$ in a-f and $20 \mu \mathrm{m}$ in $\mathbf{h}$ and $\mathbf{i}$.

obtain paraplegin-deficient mice, we employed a classical gene targeting strategy to generate a null allele (See Figure 1, a and b, and Methods for details). Spg $7^{-1-}$ mice were delivered in expected mendelian ratios and were viable. To verify that the targeted genetic disruption of Spg7 resulted in a null allele, we performed RT-PCR experiments and Western blot analyses and confirmed that neither $S p g 7 \mathrm{mRNA}$ nor paraplegin protein is present in $S p g 7^{-/-}$mice (Figure 1, c and d).

At birth, $S p g 7^{-/-}$mice are indistinguishable from heterozygous or WT littermates. These mice develop normally and at weaning show size, weight, and a home cage behavior grossly comparable to that of control littermates. At 12 months of age, Spg $7^{-/-}$mice display a statistically significant loss of body weight compared with heterozygous littermates (Figure 2a). Spg $7^{-/-}$mice older than 17 months have a pronounced scoliosis and an abnormal gait characterized by uncoordinated movement of the hindlimbs. To reveal the first signs of motor impairment, Spg $7^{-/-}$and heterozygous littermates were subjected to rotarod analysis at different ages. All mice showed learning of the rotarod test as measured by an increase in the mean duration time for mice to maintain balance on the rotarod in successive trials at 2 months (Figure 2b). Spg $7^{-/-}$mice started to have difficulty maintaining balance on the rotarod at 4 months of age (Figure 2c) and showed a progressive decline in rotarod performance over the ensuing months compared with $S p g 7^{7^{+-}}$mice (Figure 2, d-f).
Paraplegin-deficient mice develop retrograde degeneration of long spinal axons, optic nerves, and sciatic nerves. We asked whether impaired motor performance in $S p g 7^{-/}$mice could be due to the degeneration of the same spinal tracts that occurs in the human disease. Semithin sections of the spinal cord of $S p g 7^{-1-}$ mice were found to be normal up to 8 months of age, when a few axonal swellings were detected in the white matter of the spinal cord (Figure 3, a and b; Table 1). Increasing numbers of axonal swellings were seen at approximately 1 year of age in association with slow, progressive loss of fibers due to degeneration. Axonal degeneration was prominent only at 15 months and in older animals (Figure 3, c-e, Table 1), indicating that the neurologic impairment of paraplegin-deficient mice observed earlier is not directly due to the loss of axons.

At the cervical level, swollen and degenerating axons predominantly affect the most medial part of the dorsal funiculi, where distal axons of sensory neurons ascend in the fasciculus gracilis (Figure 3d, Table 1). In the lumbar spinal cord, the majority of neuropathologic abnormalities are scattered into the external regions of the lateral and anterior funiculi (Table 1). These fibers likely represent long descending projections from the red nucleus, the lateral vestibular nucleus, the pontine reticular formation, the locus coeruleus, the raphe nucleus, and the nucleus retroambiguus (21). These extrapyramidal motor axons are responsible for the major motor control in rodents (21). The corticospinal 
Table 2

Morphometric analysis of ultrastructural mitochondrial changes in the anterolateral funiculi of the lumbar spinal cord

\begin{tabular}{|c|c|c|c|c|c|}
\hline Age & Genotype & $n$ & $\%$ of fibers containing abnormal mitochondria & Type of mitochondrial abnormality & Fibers scored \\
\hline \multirow[t]{2}{*}{4.5 months } & $+/+$ & 2 & $1.4 \pm 1.1$ & Hypertrophic mitochondria & 952 \\
\hline & $-1-$ & 2 & $11.4 \pm 2$ & & 665 \\
\hline \multirow[t]{5}{*}{8 months } & $+/+$ & 2 & $0.8 \pm 0.4$ & Hypertrophic mitochondria & 991 \\
\hline & $-/-$ & 2 & $20 \pm 1.4$ & Concentric cristae & 1,135 \\
\hline & & & & Mitochondrial "bridges" & \\
\hline & & & & Giant mitochondria & \\
\hline & & & & Swollen mitochondria & \\
\hline \multirow[t]{2}{*}{15 months } & $+/+$ & 1 & 2.9 & Swollen mitochondria & 377 \\
\hline & $-1-$ & 3 & $36.3 \pm 2.8$ & & 1,093 \\
\hline
\end{tabular}

tract is affected only in very old $S p g 7^{-1-}$ mice (29 months, Table 1). However, the murine corticospinal tract descends in the ventralmost part of the dorsal funiculus and is mainly polysynaptic, being composed of shorter axons (22). Table 1 shows quantification, distribution, and progression of the axonal lesions. The topography of affected fibers and the gradient of severity of the neuropathology from distal to proximal along the axon length strongly indicate retrograde axonal degeneration, as occurs in human HSP patients.

Most patients with SPG7 mutations develop a complicated form of spastic paraplegia. Peripheral neuropathy, optic atrophy, and slight muscular involvement have been reported in a subset of patients $(7,12)$. To test for the presence of these complicated clinical features, we analyzed the optic nerves, sciatic nerves, and skeletal muscles of paraplegin-deficient and control animals at different ages. We found involvement of the optic nerve in $S p g 7^{-1-}$ mice of 19 months of age. Axonal swellings were detected close to the optic chiasm in the central region of the nerve (Figure 3f). However, degenerating axons were not identified, indicating that the phenotype in the optic nerve arises later and is milder than in spinal axons. A progressive axonopathy was also detected in the sciatic nerve. We could detect the very first sign of peripheral axon involvement in the most distal sensory terminations at 8 months of age with the appearance of axonal spheroids, intensively positive for neurofilament, in the derma of the distal hindlimbs (Figure 3g). Few axoplasmic alterations were present at 15 months of age in the distal sciatic nerve, and signs of degeneration appeared at 19 months and became prominent only at very old ages (Figure 3, h and i). Notably, ventral roots were found to be normal even at 29 months of age, strongly indicating a distal neuropathy.

Variability of fiber diameters with type I fiber hypotrophy, angulated fibers, some centrally placed nuclei, and fiber splitting were observed in the skeletal muscle of Spg $7^{-/-}$mice starting at 13 months of age (Figure 4). This phenotype worsens with age, and is complicated by the occurrence of targetoid changes at 18 months of age (Figure 4d). These features suggest changes due to denervation and reinnervation processes secondary to the peripheral neuropathy.
These data indicate that, in mouse as in man, paraplegin deficiency causes a distal axonopathy involving preferentially long axons of both the central and peripheral nervous systems.

Abnormal mitochondria appear in distal regions of affected axons long before swelling and degeneration. The first axonal swellings arise in the spinal cord of $S p g 7^{-/-}$mice at 8 months of age. However, these mice showed a decrease in their motor ability 4 months earlier (Figure 2b, Table 1 ), suggesting that axonal dysfunction precedes any morphological damage to the axon. To detect the first signs of pathologic alterations and not those secondary to axonal degeneration, we performed detailed electron microscopy analyses on spinal cord sections of paraplegin-deficient and control mice starting at 4.5 months of age (Figure 5). All these studies were performed at lumbar levels and focused on the anterolateral funiculi, where the distal regions of descending axons are located. Axons of the dorsal columns, which are never affected at the lumbar level (Table 1), allowed
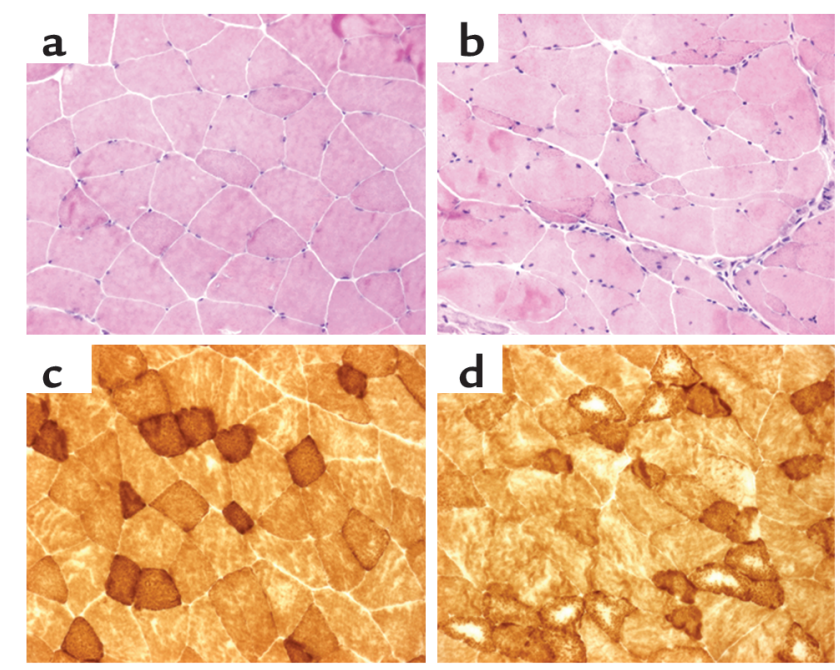

\section{Figure 4}

Muscular phenotype in paraplegin-deficient mice. H\&E and cytochrome $c$ oxidase staining of the skeletal muscle of a control (a and $\mathbf{c}$ ) and a knockout mouse (b and $\mathbf{d}$ ) at 13.5 months showing fiber hypotrophy, splitting, angulated fibers, central nuclei (b), and targetoid cores $(\mathbf{d})$ in $S p g 7^{-/-}$mice. 


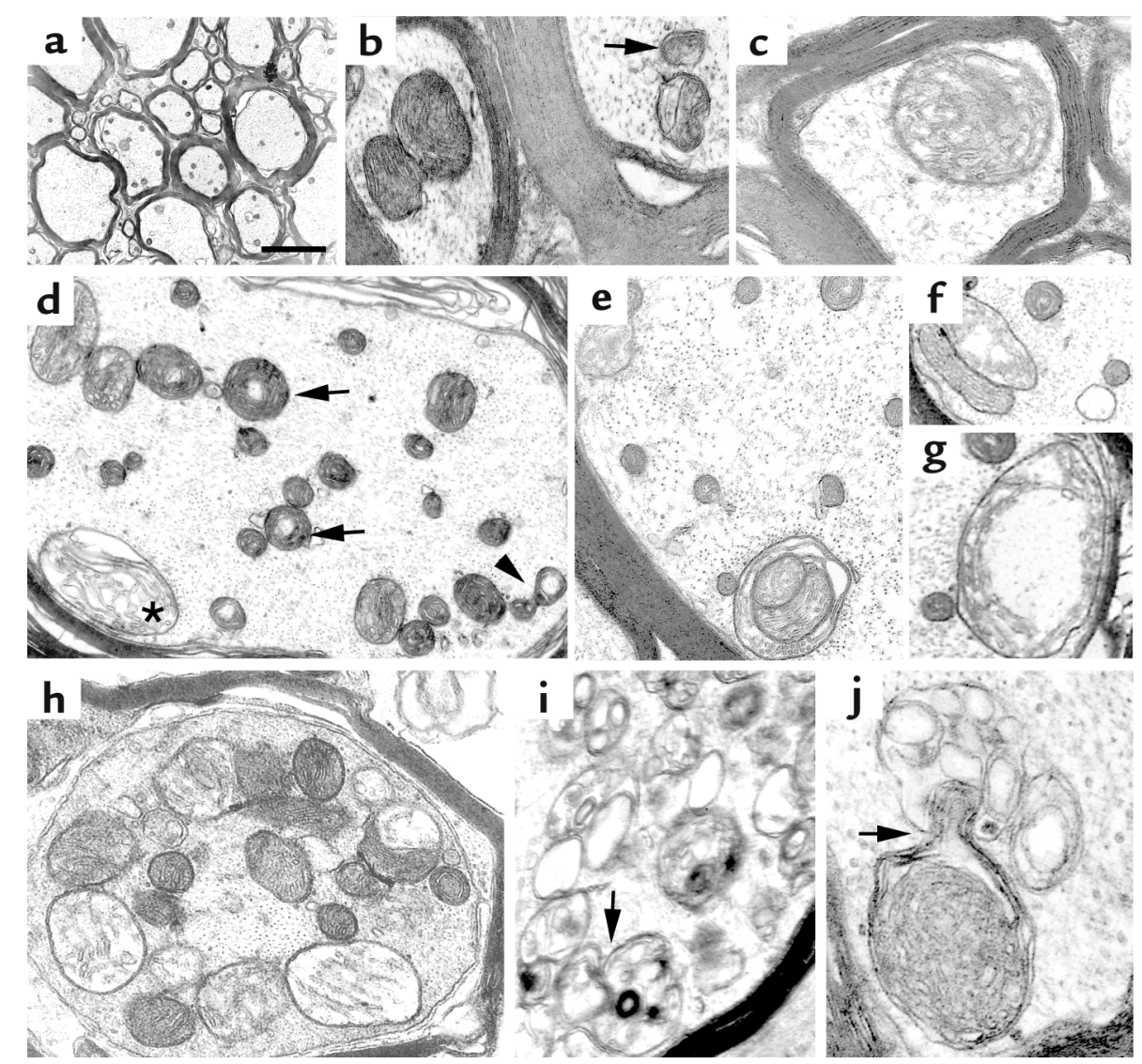

Figure 5

Mitochondrial abnormalities in paraplegin-deficient mice. (a) The spinal cord has an overall normal structure in a 4.5-month-old Spg7-/mouse, but high magnification analysis shows hypertrophic mitochondria (b), sometimes with swollen, disorganized cristae (arrow) (c). Several mitochondrial abnormalities are identifiable in the spinal cord at 8 months of age: swollen mitochondria with cristae still readily identifiable (asterisk), onion-like arrangement of mitochondrial cristae (arrows) (d), gigantic mitochondrion with abnormally organized membrane compartments and abnormal tubular content (e), closely associated mitochondria (f), and swollen mitochondria in which cristae are displaced at the periphery of the organelle ( $\mathbf{g}$ ). (h) Spinal axon of a 12-month-old Spg $7^{-/-}$mouse containing degenerating mitochondria. (i) At 15 months of age, mitochondria are swollen and show degenerative features. There is a drastic reduction in the number of cristae, which are distended. The deposition of dense deposits can be observed. Mitochondrial bridges are also evident (arrow). (j) Transverse section of the optic nerve of 22-month-old paraplegin-deficient mouse showing one hypertrophic mitochondrion extending a finger-like protrusion (mitochondrial "bridge"; arrow) into a neighboring mitochondrion, which shows features of swelling and degeneration. Bar represents $5 \mu \mathrm{m}$ in $\mathbf{a}, 0.8 \mu \mathrm{m}$ in $\mathbf{b}, \mathbf{c}, \mathbf{f}, \mathbf{g}$, and $\mathbf{j}$, and $1 \mu \mathrm{m}$ in $\mathbf{d}, \mathbf{e}, \mathbf{h}$, and $\mathbf{i}$.

us to examine proximal axons in the same section and provided an internal control to exclude artifacts resulting from tissue processing and fixation.

At 4.5 months, the ultrastructure of the spinal cord appeared normal overall (Figure 5a). However, careful examination revealed that a consistent percentage of axons in the anterolateral funiculi contain hypertrophic mitochondria (Table 2, Figure 5b). Mitochondrial hypertrophy is easily distinguishable from mitochondrial degeneration, because there is no reduction of matrix density, and the concentration of cristae is normal or increased. Isolated cases of mitochondria with swollen cristae were observed (Figure 5c). At 8 months of age, although only an extremely low number of axonal swellings/section were found in the anterolateral funiculi of $S p g 7^{-/}$mice at the lumbar level (Table 1), electron microscopy revealed that approximately $20 \% \pm 1.4 \%$ of axons of the same tracts were filled with mitochondria that were abnormal in size and morphology. Besides hypertrophic mitochondria, we could distinguish mitochondria with concentric cristae, mitochondrial herniations or "bridges," and giant mitochondria (Table 2, Figure 5, d-g). Concentric cristae are believed to occur when metabolic activity is increased in normal or pathologic situations (23). Mitochondrial bridges represent herniations of a finger- or knob-like structure from one mitochondrion to a neighboring one (23). It has been suggested that coalescence of mitochondria may occur in this way. Interestingly, we also observed a significant number of giant mitochondria at this age. It is difficult to establish whether these mitochondria derive from fusion of several mitochondria or through the enlargement of a single mitochondrion. Giant mitochondria often showed alterations of the internal structure, with novel arrangements of the membranes 

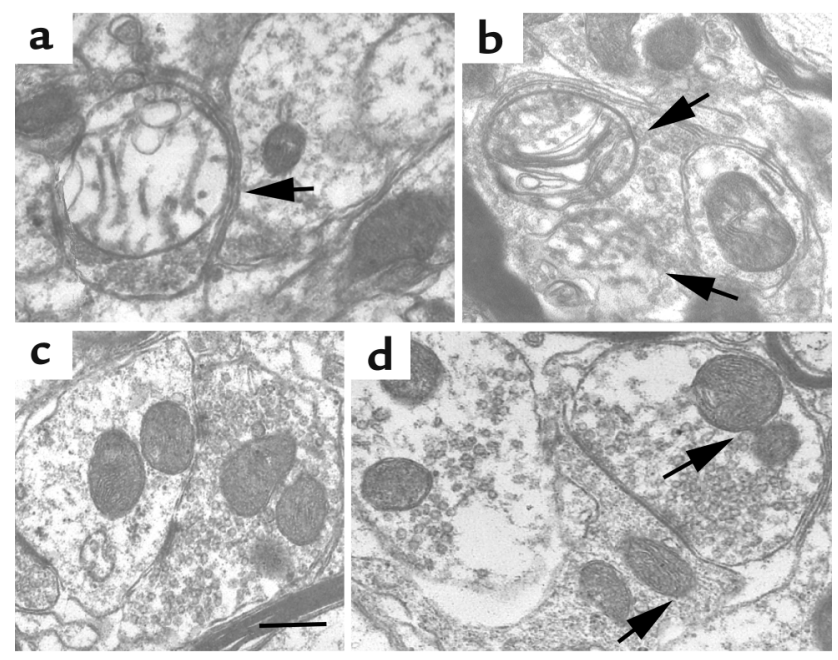

Figure 6

Early mitochondrial abnormalities in synaptic terminals of paraplegin-deficient mice. (a and $\mathbf{b}$ ) Cross sections of the gray matter of the lumbar spinal cord from 4.5-month-old Spg $7^{-1-}$ mice showing nerve terminals. Enlarged mitochondria with indistinct and damaged cristae are present (arrows). (c and $\mathbf{d}$ ) Comparable sections from age-matched control mice show typical terminal profiles with many synaptic vesicles and normal mitochondria (arrows). Bar: $0.5 \mu \mathrm{m}$.

and cristae (Figure 5e). At this age (8 months), we also noticed the first signs of mitochondrial degeneration. Several mitochondria showed a focal separation between the outer and inner mitochondrial membrane, and in a few cases mitochondrial swelling was observed. Grossly swollen hydropic mitochondria with disrupted cristae were prominent at 15 months of age (Figure 5, h and i). Mitochondrial swelling is an unspecific finding common to several pathologic conditions (23), and likely represents the final stage of mitochondrial dysfunction in affected axons.

At each age analyzed, the percentage of axons containing abnormal mitochondria greatly exceeded the number of swollen or degenerated axons. Furthermore, all the ultrastructural abnormalities found in the lumbar spinal cord are characteristically confined to the anterolateral funiculi and never detected in the dorsal columns, indicating that they (a) are not due to artifacts, (b) correlate with the topographic distribution of axonal changes, and (c) most interestingly, are specific to distal regions of axons. This latter observation prompted us to search for mitochondrial changes in synaptic terminals. We found that structurally abnormal, swollen mitochondria are already present in synaptic terminals of paraplegin-deficient mice in the anterior horn of the lumbar spinal cord at 4.5 months of age (see Figure 6), indicating that mitochondrial dysfunction in nerve terminals is the earliest pathologic event in paraplegin-deficient mice and correlates with the appearance of the neurologic phenotype.

Mitochondrial abnormalities similar to those described in the spinal cord were also found in the optic (Figure $5 \mathrm{j}$ ) and sciatic nerves in both myelinated and nonmyelinated fibers (see Figure 8, d and e). Notably, mitochondria were always found to be normal in Schwann cells surrounding affected axons, again emphasizing the axonal specificity of the phenotype (see Figure $8, \mathrm{~d}$ and $\mathrm{e}$ ). We found a certain increase in the number of subsarcolemmal mitochondria in the skeletal muscle of mice older than 24 months. In a few cases, mitochondria were swollen and contained glycogen and membrane whorls (our unpublished data). However, in the muscle, the mitochondrial phenotype is much less evident than in central and peripheral axons.

To further investigate the role of mitochondrial dysfunction in the pathogenesis of HSP due to lack of paraplegin, we studied the enzymatic activities of the respiratory chain in mitochondria isolated from different tissues of paraplegin-deficient mice and control littermates. Biochemical analyses of brain, spinal cord, and muscle of 17- to 20-month-old Spg $7^{-1-}$ and control littermates did not reveal statistically significant deficiency of complexes I, II, III, and IV (not shown). In Spg $7^{-1-}$ mice of 26-30 months, we found a tendency to a reduction of complex I activity in mitochondria isolated from the spinal cord (complex I activity/citrate synthase activity: $0.3 \pm 0.04$ in $S p g 7^{+/+}$mice versus $0.2 \pm 0.07$ in $S p g 7^{-/-}$mice; $n=3$ for $S p g 7^{+/+}$and $n=6$ for $S p g 7^{-/}$ mice; $P=0.04$ by two-tailed unpaired $t$ test with unequal variances). These data prompted us to investigate the ability of mitochondria isolated from the spinal cord of Spg $7^{-/-}$mice to synthesize ATP. ATP synthesis was measured using a luciferase-luciferin assay after delivering substrates to freshly prepared mitochondria isolated from the spinal cord of paraplegindeficient and control mice of 7, 17, and 23-26 months. We found comparable levels of ATP at 7 and 17 months of age in paraplegin-deficient mice compared with WT littermates (Figure 7). A reduction of approximately $15 \%$ of ATP synthesis was evident only at 23-26 months of age in $\operatorname{Spg} 7^{--}$mice (Figure 7).

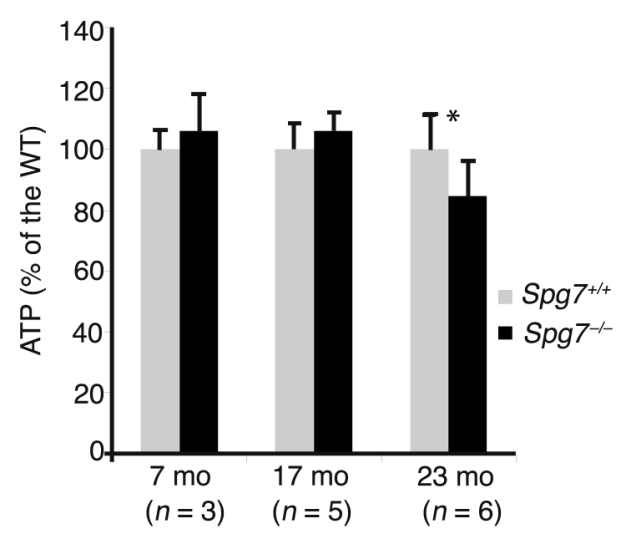

\section{Figure 7}

ATP synthesis in mitochondria isolated from the spinal cord of WT and $S p g 7^{-1-}$ mice in the presence of pyruvate and L-malate as substrates. Values are expressed as percentage of the controls. Bars show $\mathrm{SEM} ; n$ indicates the number of independent experiments performed at each age (see Methods). ${ }^{*} P<0.02$. 


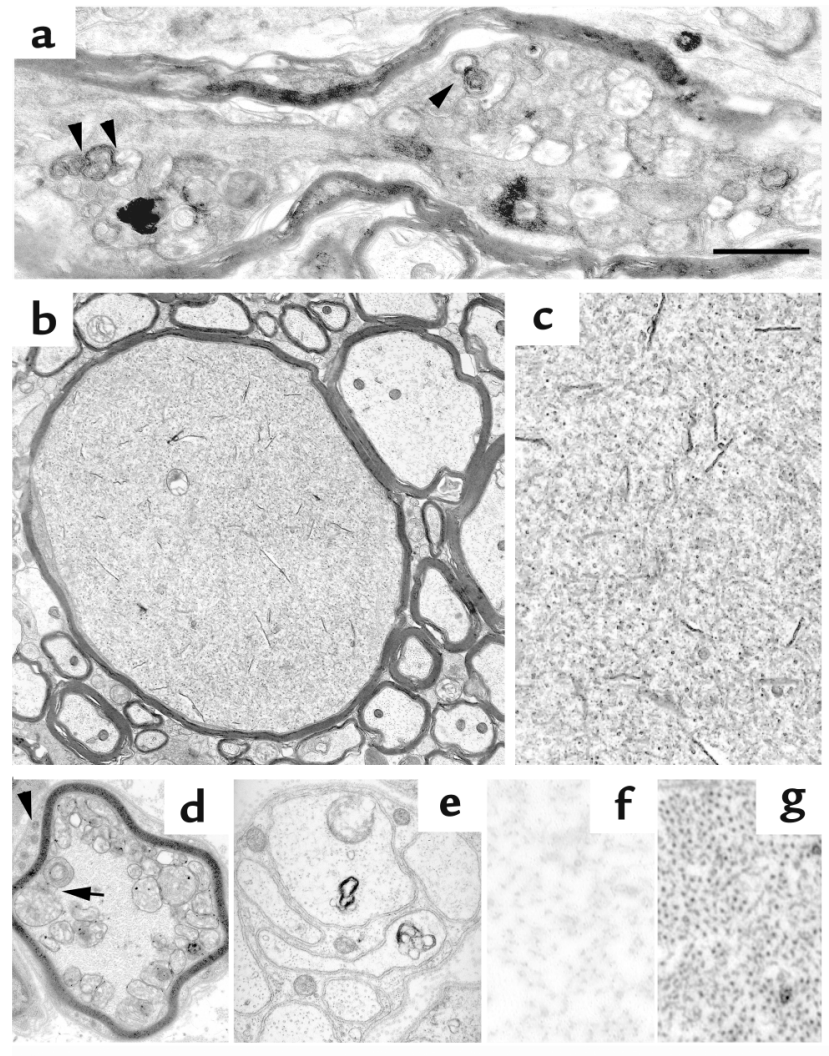

These data further emphasize that paraplegin deficiency affects only a specific subset of mitochondria while allowing normal ATP synthesis and oxidative phosphorylation in most tissues. It is not surprising that defective ATP synthesis and reduction of complex I activity are measurable only at late stages of the disease, since in the spinal cord, abnormal mitochondria are confined to the distal region of only a subset of axons (see above) and therefore represent only a small percentage of the total mitochondria isolated from an entire spinal cord. Future biochemical studies will require specific analysis of distinct subpopulations of mitochondria from affected axons.

Neurofilaments accumulate in affected axons. Ultrastructural analysis of swollen axons in paraplegin-deficient mice shows that axonal spheroids represent segmental distensions of the axons containing an accumulation of neurofilaments and organelles (Figure 8). Enlarged spinal axons are filled with numerous dense and multivesicular bodies and electron-dense amorphous material (Figure 8a). In several cases, affected axons contain a high number of abnormal or swollen mitochondria (Figure 8a and Figure 5). In other cases, axonal swellings occur because of massive accumulation of filamentous aggregates (Figure 8b). In these axons, neurofilaments are closely packed and unevenly distributed, in contrast with the regularly spaced, parallel arrays of neurofilaments in unaffected axons (Figure 8c). In many instances, bundles of filaments did not extend parallel to the long axis of the axon (Figure 8c). Similar ultrastructural features are found in the

\section{Figure 8}

Ultrastructural analyses of axons in the spinal cord and the sciatic nerve of $S p g 7^{-1-}$ mice show accumulation of organelles and disorganization of the cytoskeleton. (a) Longitudinal section of a spinal axon of a 15-month-old paraplegin-deficient mouse, showing segmental swellings. Filamentous aggregates and degenerating mitochondria (arrows) accumulate in the axoplasm. (b) Transverse section of the spinal cord in a 15 -month-old Spg $7^{-/-}$mouse shows an axonal swelling with massive accumulation of filamentous aggregates and loss of organelles. (c) Enlargement of the axoplasm of the axon in $\mathbf{b}$ showing that neurofilaments have lost their normal orientation and form disorganized bundles. ( $\mathbf{d}$ and $\mathbf{e}$ ) Electron microscopy analysis of sciatic nerve of a 22-month-old Spg $7^{-/-}$mouse. Neurofilamentous accumulations and focal accumulations of enlarged mitochondria with varying degrees of loss of structural integrity, and excessive or disorganized cristae, are observed in myelinated fibers (d). Arrow in $\mathbf{d}$ indicates onion-like arrangement of mitochondrial cristae. Abnormal mitochondria are present also in unmyelinated fibers (e). Both myelin-forming and non-myelin-forming Schwann cells show normal mitochondria (arrowhead in $\mathbf{d}$ and $\mathbf{e}$ ). ( $\mathbf{f}$ and $\mathbf{g}$ ) Representative cross section of sciatic nerve axons from a $\operatorname{Spg} 7^{+/+}(\mathbf{f})$ and an $S p g 7^{-/-}(\mathbf{g})$ mouse at 22 months, showing increased neurofilament density in $S p g 7^{-1-}$ animals. Bar represents $4 \mu \mathrm{m}$ in a, $7 \mu \mathrm{m}$ in b, 0.4 $\mu \mathrm{m}$ in $\mathbf{c}, \mathbf{f}$, and $\mathbf{g}, 5 \mu \mathrm{m}$ in $\mathbf{d}$, and $3.33 \mu \mathrm{m}$ in $\mathbf{e}$.

optic (not shown) and sciatic nerves (Figure 8, d-g). Since sciatic axons show a relatively uniform axoplasmic density, we could perform a quantitative evaluation of ultrastructural changes affecting cytoskeletal elements. We analyzed randomly chosen axons in matched sections of the distal sciatic nerve of Spg $7^{-1}$ and WT mice at 19 months of age (see Methods for details). We found an evident increase in neurofilament density per given area of an axon in $S p g 7^{-/-}$mice $\left(206.9 \pm 23.3\right.$ neurofilaments $/ \mu \mathrm{m}^{2}$ in $S p g 7^{-/-}$axons vs. $83.6 \pm 7.9$ neurofilaments $/ \mu \mathrm{m}^{2}$ in WT axons; $n=6$; $\left.P=2.3 \times 10^{-7}\right)$. The presence of focal axonal swellings that accumulate neurofilaments and organelles is highly suggestive of an impairment of both slow and fast anterograde axonal transport.

Delay of retrograde transport in paraplegin-deficient mice. To investigate the possibility that retrograde transport is also affected in paraplegin-deficient mice, we performed neurotracer studies in the peripheral nerve. We injected cholera toxin subunit B conjugated with a fluorescent dye into the gastrocnemius muscle of paraplegin-deficient and age-matched heterozygous control mice. The B subunit of cholera toxin has proven to be a powerful tool for retrograde labeling of neurons (24). Animals were sacrificed 48 hours later to determine the number of labeled motor neurons. No significant differences were found in young, presymptomatic animals (not shown). In mice 17 months of age, we detected a slight but statistically significant reduction of the total number of labeled motor neurons in paraplegin-deficient mice compared with control animals (Figure 9a). This reduction is restricted to motor neurons located in the upper third of the lumbar enlargement, suggesting that paraplegin-deficient mice suffer from a delay in retrograde transport that affects neurons with the 


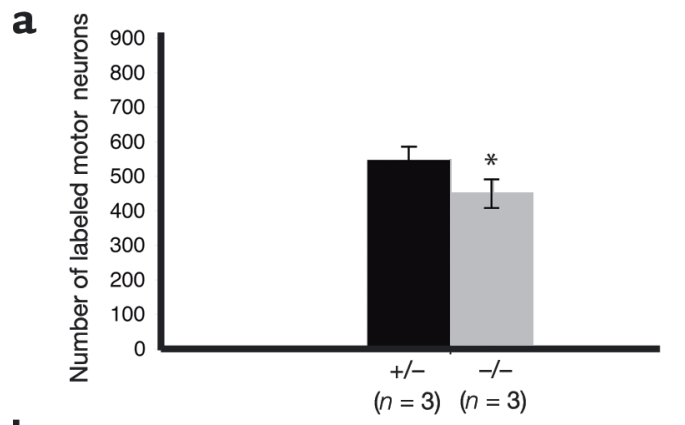

b
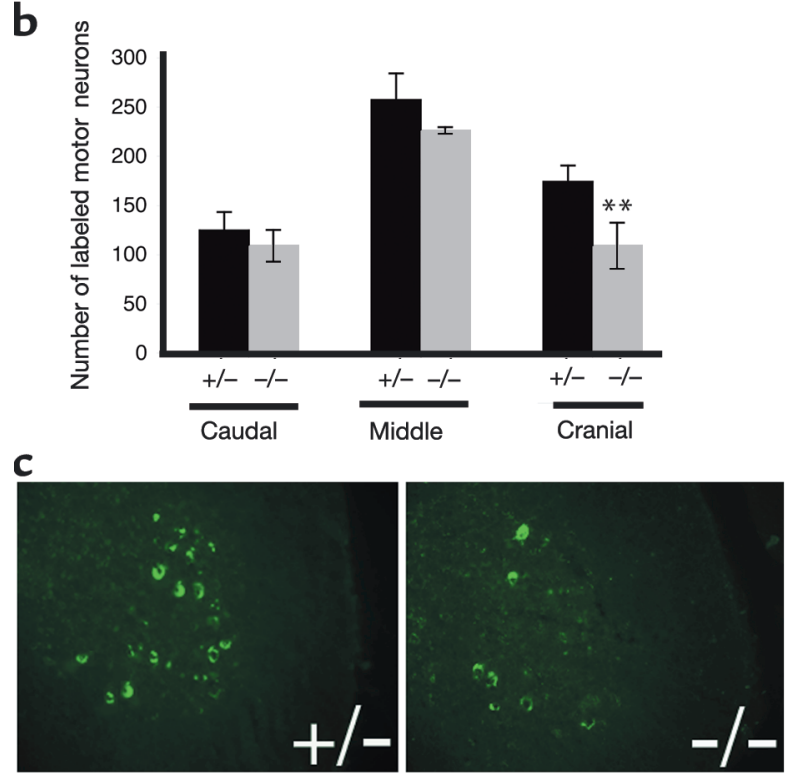

Figure 9

Delay of retrograde transport in paraplegin-deficient mice. (a) The total number of labeled motor neurons in the lumbar enlargement 48 hours after injection of fluorescent cholera toxin subunit B in the gastrocnemius is reduced in $S p g 7^{-1-}$ mice compared with heterozygous control mice. (b) Quantification of the number of labeled motor neurons in the cranial, middle, and caudal regions of the lumbar enlargement shows that the reduction in the number of labeled motor neurons is confined to the cranial region, where paraplegin-deficient mice have approximately $38 \%$ fewer labeled motor neurons than controls have. (c) Transverse sections of the spinal cord of Spg $7^{-/-}$and Spg $7^{+/-}$ mice in the cranial region of the lumbar enlargement showing the decrease in the number of labeled motor neurons in paraplegin-deficient mice compared with controls. ${ }^{*} P<0.05 ;{ }^{*} P<0.01$.

longest axons and can be compensated for by allowing enough time for the fluorescent tracer to reach the target cell (Figure 9, b and c).

\section{Discussion}

Very little is known about the mechanisms through which corticospinal axons degenerate in HSP, although numerous genes responsible for this disease have been identified in recent years (25). We contribute here to the understanding of the pathogenic cascade underlying axonal degeneration by studying a mouse model for autosomal recessive HSP due to loss-offunction mutations in the SPG7 gene. Paraplegin-deficient mice are affected by a progressive retrograde degeneration of long ascending and descending tracts in the spinal cord and by optic neuropathy, peripheral axonopathy, and a mild muscular involvement. We provide evidence that axonal degeneration begins distally and then slowly moves proximally along the axon. We also show that axonal length is an important determinant for the selective involvement of a subset of axons in HSP. In the mouse, the corticospinal tract, being mainly polysynaptic, is composed of shorter axons (22). Consistently its axons degenerate only in very old animals, in contrast with what occurs in humans. Finally, our findings indicate that paraplegin deficiency causes a disease mainly of axons. In both human and mouse, involvement of the skeletal muscle is very mild and in the mouse is largely dependent on the peripheral neuropathy.

The first ultrastructural feature that distinguishes the spinal cord of paraplegin-deficient mice from that of control littermates occurs at about 4.5 months of age and is represented by the appearance of abnormal mitochondria in synaptic terminals in the gray matter of the lumbar spinal cord. At this age, hypertrophic mitochondria are also present in a relevant percentage of long axons, distal to their cellular bodies. Notably, this mitochondrial phenotype correlates with the onset of a significant motor deficit on the rotarod and occurs several months prior to axonal degeneration, suggesting that it is the primary cause for axonal dysfunction and that the neurologic impairment of paraplegin-deficient mice is not directly the result of the loss of axons. This latter observation implies that there is a temporal window during which therapeutic intervention could actually prevent loss of axons.

Nerve terminals invariably contain many mitochondria, which are involved in ATP production, in regulation of cellular calcium homeostasis, and in oxyradical metabolism. All these functions may be affected in paraplegin-deficient mice and contribute to the early axonal dysfunction. One technical problem to address this issue comes from the very limited number of affected mitochondria in the spinal cord and from the inherent complexity of purifying them. We were able to measure reduced ATP synthesis in the spinal cord of very old Spg $7^{-/-}$mice, suggesting that defective ATP production may play a role in the pathogenesis of HSP, at least in late stages of the disease. The establishment of neuronal cell cultures from paraplegin-deficient mice will allow measurement of the mitochondrial membrane potential and investigation of the mechanisms of mitochondrial dysfunction in the future.

With aging, there is an increase in the percentage of axons showing mitochondrial abnormalities in paraplegin-deficient mice. Furthermore, mitochondria undergo other significant changes, such as rearrangements of cristae, formation of bridges with adjacent mitochondria, and acquisition of giant dimensions. These data raise three important questions: Are the mitochondrial structural abnormalities a primary or a secondary effect of the lack of paraplegin? Why does 
this phenotype selectively involve only a subset of mitochondria, given the ubiquitous expression pattern of paraplegin? Finally, what is the link between the mitochondrial phenotype and the axonal dysfunction?

Paraplegin is one of the mammalian orthologues of the yeast m-AAA protease, whose role in proteolytic degradation of inner mitochondrial membrane proteins and in regulation of mitochondrial biogenesis has been extensively documented (11). On the basis of this knowledge, structurally abnormal mitochondria in $S p g 7^{-1-}$ mice may be the result of the accumulation of abnormal, misfolded proteins in the inner mitochondrial membrane. Alternatively, the abnormal mitochondria could result from impaired turnover or processing of a regulatory protein involved in some aspect of mitochondrial dynamics. Mitochondrial morphology is in fact regulated by the balance between processes of fusion and fission, and many extrinsic factors, including proteases, may influence and contribute to this plasticity (26-28). The identification of paraplegin substrates will provide important hints to confirm or reject these hypotheses. Finally, we cannot exclude that the abnormal and specialized mitochondrial shapes that we observe in our mice may just be secondary to impaired respiration and serve to increase the ratio of surface area to volume. However, we do not favor this possibility, because in paraplegindeficient mice, mitochondrial swelling occurs a long time after the first morphological abnormalities are detected and is preceded by a tremendous increase in the size of the organelle.

An interesting and puzzling finding is that, although paraplegin is a ubiquitous protein, abnormalities are restricted only to a subset of mitochondria. Recent evidence suggests that mitochondria are highly dynamic organelles whose shape and function are regulated during growth and development and are dependent on cellular and tissue metabolic demands (29). For example, mitofusin-2, one of the molecules involved in mammalian mitochondrial fusion events, is required only for selective developmental transitions in the mouse, despite a broad pattern of expression (30). The morphological and functional heterogeneity of mitochondria raises the possibility that subpopulations of these organelles play different roles within different areas of a cell (31). Neurons are highly specialized and polarized cells with processes that can reach a length of up to 1 meter in humans. Since the slow diffusion of ATP could limit their metabolic function, neurons meet their energy requirements by placing mitochondria near the site of demand. Accordingly, the appropriate regulation of transport and distribution of mitochondria is an essential component of the life and function of a neuron (32). A clear understanding of the life cycle of mitochondria in postmitotic cells such as neurons is currently missing. The pattern of mitochondrial motility in axons includes bidirectional saltatory movement and prolonged stationary phases (32). Mitochondria located very distal to the neural cell body could be required to endure for longer periods of time, and could be much less efficient in adapting to a lack of paraplegin through the recruitment of molecules involved in handling misfolded proteins or in upregulating expression of the respiratory chain components. Furthermore, regulation of mitochondrial morphology in neurons and in different regions of axons may also play a key role. Interestingly, the optic atrophy type I gene (OPA1), the orthologue of the yeast MGM1 gene involved in regulating mitochondrial morphology, is mutated in a dominantly inherited optic atrophy (33-35).

The appearance of structurally abnormal mitochondria in affected axons of paraplegin-deficient mice is followed in time by segmental swelling due to accumulation of neurofilaments and organelles. This finding strongly suggests impairment of anterograde axonal transport. Axonal transport is an energydependent process that is especially crucial to the long axons affected in HSP, which transport molecules and organelles assembled in the neuronal cell body across long distances. Disorganization of the neurofilament network is a common hallmark of toxic neuropathies (36) and of many neurodegenerative diseases, such as amyotrophic lateral sclerosis, giant axonal neuropathy, infantile spinal muscular atrophy, and axonal Charcot-Marie-Tooth disease (37-42). Axonal inclusions of intermediate filaments have been proposed to "strangulate" the axon by a chronic deficit in transport, thus leading to neurodegeneration (43). In fact, neurofilament accumulation itself may have a toxic effect by further affecting slow axonal transport (44) and sequestering mitochondria and other organelles. Changes in neurofilament levels also perturb the dynamics and function of microtubules, which are key organelles of intracellular transport.

We found evidence that retrograde axonal transport is delayed in paraplegin-deficient mice. This defect was found only in symptomatic mice, suggesting that impairment of axonal transport is not a primary, but rather a secondary effect. However, impairment of retrograde transport could contribute significantly to the ultimate degeneration of axons by affecting the transport of mitochondria, the trafficking of endosomes, and the internalization of neurotrophic factors. Disruption of retrograde axonal transport has already been shown to cause progressive degeneration of motor neurons in both mice and humans $(45,46)$.

Aberrant cellular trafficking dynamics has been suggested to underlie several other forms of HSP, based on the predicted function of the genes involved (reviewed in ref. 25). Although involvement of HSP genes in axonal transport is purely speculative in most cases, autosomal dominant HSP linked to chromosome $12 \mathrm{q}$, $S P G 10$, is due to mutations in a kinesin heavy chain gene, KIF5A (47). Furthermore, mice lacking proteolipid protein 1 (PLP1), the only other mouse model available for HSP, display axonal degeneration associated with the accumulation of neurofilaments, mito- 
chondria, and other organelles (48). These data, together with our findings in paraplegin-deficient mice, suggest that chronic reduction of axonal transport is a common pathway through which axons degenerate in many forms of HSP, prompting us to search for therapeutic agents that would ameliorate it, with the notion that a timely intervention may indeed be successful in preventing axonal degeneration.

\section{Acknowledgments}

The authors wish to thank Emanuele Marra for technical assistance and Alessia Errico, Luigia Atorino, Laura Silvestri, Graciana Diez-Roux, Valeria Marigo, Germana Meroni, Lawrence Wrabetz, and Thomas Langer for helpful discussion and critical reading of the manuscript. This work was supported by grants from the Italian Telethon Foundation, the Muscular Dystrophy Association, and the Italian Ministry of Health to E.I. Rugarli and from the NIH (R01 NS-38713-01) to A. Ballabio. F. Ferreirinha has a graduate fellowship (PRAXIS XXI/BD/19984/99) from the Fundação para a Ciência e a Tecnologia-Portugal.

1. Kobayashi, H., Hoffman, E.P., and Marks, H.G. 1994. The rumpshaker mutation in spastic paraplegia. Nat. Genet. 7:351-352.

2. Fink, J.K., et al. 1996. Hereditary spastic paraplegia: advances in genetic research. Neurology. 46:1507-1514.

3. Harding, A.E. 1983. Classification of the hereditary ataxias and paraplegias. Lancet. 1151-1154.

4. Fink, J.K. 1997. Advances in hereditary spastic paraplegia. Curr. Opin. Neurol. 10:313-318.

5. Harding, A.E. 1984. Hereditary "pure" spastic paraplegia. The hereditary ataxias and related disorders. Churchill Livingstone. New York, New York, USA. 174-191.

6. Casari, G., and Rugarli, E. 2001. Molecular basis of inherited spastic paraplegias. Curr. Opin. Genet. Dev. 11:336-342.

7. Casari, G., et al. 1998. Spastic paraplegia and OXPHOS impairment caused by mutations in paraplegin, a nuclear-encoded mitochondrial metalloprotease. Cell. 93:973-983.

8. Arlt, H., Tauer, R., Feldmann, H., Neupert, W., and Langer, T. 1996. The YTA10-12 complex, an AAA protease with chaperone-like activity in the inner membrane of mitochondria. Cell. 85:875-885.

9. Confalonieri, F., and Duguet, M. 1995. A 200-amino acid ATPase module in search of a basic function. Bioessays. 17:639-650.

10. Neuwald, A.F., Aravind, L., Spouge, J.L., and Koonin, E.V. 1999. AAA+: a class of chaperone-like ATPases associated with the assembly, operation, and disassembly of protein complexes. Genome Res. 9:27-43.

11. Langer, T., Kaser, M., Klanner, C., and Leonhard, K. 2001. AAA proteases of mitochondria: quality control of membrane proteins and regulatory functions during mitochondrial biogenesis. Biochem. Soc. Trans. 29:431-436.

12. McDermott, C.J., et al. 2001. Paraplegin gene analysis in hereditary spastic paraparesis (HSP) pedigrees in northeast England. Neurology. 56:467-471.

13. Hansen, J.J., et al. 2002. Hereditary spastic paraplegia SPG13 is associated with a mutation in the gene encoding the mitochondrial chaperonin Hsp60. Am. J. Hum. Genet. 70:1328-1332.

14. Harlow, E., and Lane, D. 1988. Antibodies: a laboratory manual. Cold Spring Harbor Laboratory Press. Cold Spring Harbor, New York, USA. 726.

15. Rosenthal, R.E., Hamud, F., Fiskum, G., Varghese, P.J., and Sharpe, S. 1987. Cerebral ischemia and reperfusion: prevention of brain mitochondrial injury by lidoflazine. J. Cereb. Blood Flow Metab. 7:752-758.

16. Ronner, P., Friel, E., Czerniawski, K., and Frankle, S. 1999. Luminometric assays of ATP, phosphocreatine, and creatine for estimation of free ADP and free AMP. Anal. Biochem. 275:208-216.

17. Robinson, B.H., et al. 1990. The use of skin fibroblast cultures in the detection of respiratory chain defects in patients with lacticacidemia. Pediatr. Res. 28:549-555.
18. Previtali, S.C., et al. 2000. Epitope-tagged P(0) glycoprotein causes CharcotMarie-Tooth-like neuropathy in transgenic mice.J. Cell Biol. 151:1035-1046.

19. Consiglio, A., et al. 2001. In vivo gene therapy of metachromatic leukodystrophy by lentiviral vectors: correction of neuropathology and protection against learning impairments in affected mice. Nat. Med. 7:310-316.

20. Dubowitz, V. 1985. Muscle biopsy: a practical approach. Balliere Tindall. London, United Kingdom. 720 pp.

21. Nieuwenhuys, R., Ten Donkellar, H.J., and Nicholson, C. 1998. The cen tral nervous system of vertebrates. Springer-Verlag. Berlin, Germany. 1668-1672.

22. Terashima, T. 1995. Anatomy, development and lesion-induced plasticity of rodent corticospinal tract. Neurosci. Res. 22:139-161.

23. Ghadially, F.N. 1988. Ultrastructural pathology of the cell and matrix. Butterworth-Heinemann. Boston, Massachusetts, USA. 1414 pp.

24. Wan, X.C., Trojanowski, J.Q., and Gonatas, J.O. 1982. Cholera toxin and wheat germ agglutinin conjugates as neuroanatomical probes: their uptake and clearance, transganglionic and retrograde transport and sensitivity. Brain Res. 243:215-224.

25. Crosby, A.H., and Proukakis, C. 2002. Is the transportation highway the right road for hereditary spastic paraplegia? Am. J. Hum. Genet. 71:1009-1016.

26. Westermann, B. 2002. Merging mitochondria matters: cellular role and molecular machinery of mitochondrial fusion. EMBO Rep. 3:527-531.

27. Yoon, Y., and McNiven, M.A. 2001. Mitochondrial division: new partners in membrane pinching. Curr. Biol. 11:R67-R70.

28. McQuibban, G.A., Saurya, S., and Freeman, M. 2003. Mitochondrial membrane remodelling regulated by a conserved rhomboid protease. Nature. 423:537-541.

29. Yaffe, M.P. 1999. The machinery of mitochondrial inheritance and behavior. Science. 283:1493-1497.

30. Chen, H., et al. 2003. Mitofusins Mfn 1 and Mfn 2 coordinately regulate mitochondrial fusion and are essential for embryonic development. J. Cell Biol. 160:189-200.

31. Collins, T.J., Berridge, M.J., Lipp, P., and Bootman, M.D. 2002. Mitochondria are morphologically and functionally heterogeneous within cells. EMBO J. 21:1616-1627.

32. Hollenbeck, P.J. 1996. The pattern and mechanism of mitochondrial transport in axons. Front. Biosci. 1:d91-d102.

33. Shepard, K.A., and Yaffe, M.P. 1999. The yeast dynamin-like protein, Mgm1p, functions on the mitochondrial outer membrane to mediate mitochondrial inheritance. J. Cell Biol. 144:711-720.

34. Alexander, C., et al. 2000. OPA1, encoding a dynamin-related GTPase, is mutated in autosomal dominant optic atrophy linked to chromosome 3q28. Nat. Genet. 26:211-215.

35. Delettre, C., et al. 2000. Nuclear gene OPA1, encoding a mitochondrial dynamin-related protein, is mutated in dominant optic atrophy. Nat. Genet. 26:207-210.

36. Griffin, J.W., Hoffman, P.N., Clark, A.W., Carroll, P.T., and Price, D.L. 1978. Slow axonal transport of neurofilament proteins: impairment of beta,beta'-iminodipropionitrile administration. Science. 202:633-635.

37. Bomont, P., et al. 2000. The gene encoding gigaxonin, a new member of the cytoskeletal BTB/kelch repeat family, is mutated in giant axonal neuropathy. Nat. Genet. 26:370-374.

38. Julien, J.P., and Beaulieu, J.M. 2000. Cytoskeletal abnormalities in amyotrophic lateral sclerosis: beneficial or detrimental effects? J. Neurol. Sci. 180:7-14.

39. Julien, J.P. 2001. Amyotrophic lateral sclerosis: unfolding the toxicity of the misfolded. Cell. 104:581-591.

40. Lupski, J.R. 2000. Axonal Charcot-Marie-Tooth disease and the neurofilament light gene (NF-L). Am. J. Hum. Genet. 67:8-10.

41. Mersiyanova, I.V., et al. 2000. A new variant of Charcot-Marie-Tooth disease type 2 is probably the result of a mutation in the neurofilamentlight gene. Am. J. Hum. Genet. 67:37-46.

42. Xu, Z., Cork, L.C., Griffin, J.W., and Cleveland, D.W. 1993. Involvement of neurofilaments in motor neuron disease. J. Cell Sci. Suppl. 17:101-108.

43. Cleveland, D.W. 1999. From Charcot to SOD1: mechanisms of selective motor neuron death in ALS. Neuron. 24:515-520.

44. Willard, M., and Simon, C. 1983. Modulations of neurofilament axonal transport during the development of rabbit retinal ganglion cells. Cell. 35:551-559.

45. Puls, I., et al. 2003. Mutant dynactin in motor neuron disease. Nat. Genet. 33:455-456.

46. LaMonte, B.H., et al. 2002. Disruption of dynein/dynactin inhibits axonal transport in motor neurons causing late-onset progressive degeneration. Neuron. 34:715-727.

47. Reid, E., et al. 2002. A kinesin heavy chain (KIF5A) mutation in hereditary spastic paraplegia (SPG10). Am. J. Hum. Genet. 71:1189-1194.

48. Griffiths, I., et al. 1998. Axonal swellings and degeneration in mice lacking the major proteolipid of myelin. Science. 280:1610-1613. 